\title{
Prediction of Arch Dam Temperature Based on ARIMA-RTA Model
}

\author{
Sun Guozheng \\ Zhuji Water conservancy and Hydropower Bureau \\ Zhuji, P.R.China \\ qp021625@163.com
}

\author{
Zhang Zheyu \\ National Research Institute for Rural Electrification \\ Hangzhou, P.R.China \\ qp021625@126.com
}

\begin{abstract}
The analysis and prediction of arch dam temperature is very meaningful to engineering maintenance and disaster prevention. Taking the arch dam temperature monitoring data as the research object, the ARIMA-RTA combination forecasting model is improved by using the ARIMA model's higher fitting capacity and the RTA isoperimetric recursive prediction to improve the prediction length of arch dam monitoring data. As a test, the prediction model is applied to the project of "bailianya", and the result shows that the model has carefully-defined physical conception and thus brings about vast range of prospect for application due to its high precision and noise immunity.
\end{abstract}

Keywords-arch dam; temperature monitoring; prediction; ARIMA; RTA

\section{INTRODUCTION}

For overall rolling pouring, continuous rise of the Roller compacted concrete arch dam(RCC arch dam)'s construction process, there is a problem that the concrete heat capacity is poor and hydration heat accumulated in the dam internal. Under the action of internal and external constraints, large temperature stress may occur in the dam body, which will directly affect the progress of the dam and the safety of the dam. Therefore, it is very important to carry on real-time temperature monitoring and forecasting of arch dam[1,2]

The time series is an objective record of the historical behavior of the system. It contains the structural characteristics of the system and its operating rules. It is a reliable way to predict the temperature control of arch dams by extracting the effective information of the time series [3]. Nowadays, nonlinear methods such as gray theory, chaos theory and neural network have been applied to the field of time series analysis, and some achievements have been made [4]. However, these methods have a strong dependency on the length of data. When the length of monitoring data is short or the interference of the white noise exists in the data will have great influence on the prediction accuracy of these methods.

The autoregressive integrated moving average model (ARIMA) has been widely used in the field of dam monitoring
Qin Peng

Institute of Hydraulic and Environment Engineering Zhejiang University of Water Resource and Electric Power Hangzhou, P.R.China 564293983@qq.com

data prediction. The model conforms to the requirements of the model strictly to ensure the accuracy. The higher the order, the better the fitting precision. However, it is the key to model forecasting how to improve the prediction length under the premise of guaranteeing the prediction accuracy. Aiming at this problem, the ARIMA model is improved by the real-time tracing algorithm (RTA), and a combined forecasting model of ARIMA and real-time tracking algorithm (ARIMA-RTA) is established, Dam temperature control forecast to provide a reasonable and convenient new computing model.

\section{The ESTABLISHMENT OF ARIMA MODEL}

\section{A. Establishment of ARIMA Model}

The Autoregressive Integrated Moving Average Model (ARIMA) is a well-known time-series forecasting method proposed by Box and Jenkins in the early 1970s, so it is also called the Box-jenkins model, Box-Jenkins method. ARIMA( $p$, $d, q$ )is called Differential Autoregressive Moving Average Model, where AR is autoregressive, $\mathrm{p}$ is the autoregressive term, MA is the moving average, $\mathrm{q}$ is the moving average and $\mathrm{D}$ is the number of times of difference when the time series becomes stationary[5]

The modeling steps are as follows

1) The original sequence is tested for stationary, and if the sequence does not satisfy the stationary condition, it can be transformed by the difference transform (single integer order is $\mathrm{d}$, then the difference of order d) or other transformations. The paper used autocorrelation function (AC) Autocorrelation (PAC) to test the stability of time series[5,6,7]. The specific expression for calculating the autocorrelation function is:

$$
R_{k}=E\left[\left(X_{i}-\mu_{i}\right)\left(X_{i+k}-\mu_{i+k}\right)\right] / \sigma^{2}, \quad \rho_{k}=R_{k} / R_{0}
$$

Where, $E$ Is the expected value, $X_{i}$ is the random variable value for the time, $\mu_{i}$ is the expected value for the time, $\sigma^{2}$ is the variance, and $\rho_{k}$ the autocorrelation function. 
2) According to the recognition rule of the time series model, establish the corresponding model. If the autocorrelation function is truncated and the autocorrelation function is truncated, it can be concluded that the sequence is suitable for the AR model. If the partial correlation function of the stationary series is trailing and the autocorrelation function is truncated It can be concluded that the sequence is suitable for the MA model. If the partial correlation function and the autocorrelation function of the stationary series are trailing, the sequence is suitable for the ARIMA model[5,6].

3) Determine the order p and q of the ARIMA model using the minimum Aicca Information Criterion (AIC) and select as few parameters as possible in the initial estimate. AIC is the best known ARIMA model order criterion, which can effectively overcome the over-sensitive problem of the model and improve the robustness of the model. The specific expression is:

$$
A I C(k, j)=\ln \left(\sigma^{2}(k, j)\right)+\frac{2(k+j)}{N}
$$

Where $\sigma^{2}(k, j)$ is the estimate of the white noise variance $\sigma^{2}$. On the basis of the above model identification, the unknown parameters of ARIMA model are calculated by least squares estimation.

4) Using the normal distribution test to test whether the residual sequence of the model is white noise, if more than $5 \%$ of the absolute value of the sample autocorrelation function $\left|\rho_{j}\right| \geq 1.96 / \sqrt{N}$, indicating that the residual sequence is not white noise, need to re-establish the model[7,8].

5) Use the model that has passed inspection to predict and analyze the arch dam temperature control data.

\section{B. An Improved Method of ARMA Model Based on RTA Algorithm}

On the basis of the ARIMA model, this paper proposes an improved model combining with real-time tracing algorithm (RTA). The basic idea is to predict the time series $\left\{x_{i} \mid i=1: n\right\}$, Assuming that the prediction step is $\mathrm{m}$, using the monitoring values $\left\{x_{1}, x_{2}, \cdots \cdots x_{n}\right\}$ to predict $\left\{x_{n+1}, x_{n+2}, \cdots \cdots x_{n+m}\right\}$ after the $\mathrm{n}$ times, With the back of $\mathrm{m}$ measured data obtained, remove the first $m$ data $\left\{x_{1}, x_{2}, \cdots \cdots x_{m}\right\}$, With $n$ new measured data added to the original time series $\left\{x_{m+1}, x_{m+2}, \cdots \cdots x_{n+m}\right\}$, And then constitute the next step of the forecast to maintain the data Columns.

In actual ARIMA modeling, the original sequence data of the system are not always used to model, the values of parameters $p, q$ and $b$ are difference, and the prediction results of the model are different. The time series model of the appropriate parameters must be chosen. At the same time, the metabolic model of the appropriate dimension can be constructed. The newest data is added every time, the old data is eliminated and the sequence length is kept unchanged.

\section{ARIMA-RTA Combination Model Flow Chart}

The ARIMA-RTA combination model is predicted by MATLAB. The flow chart of the model is shown in Fig.1.

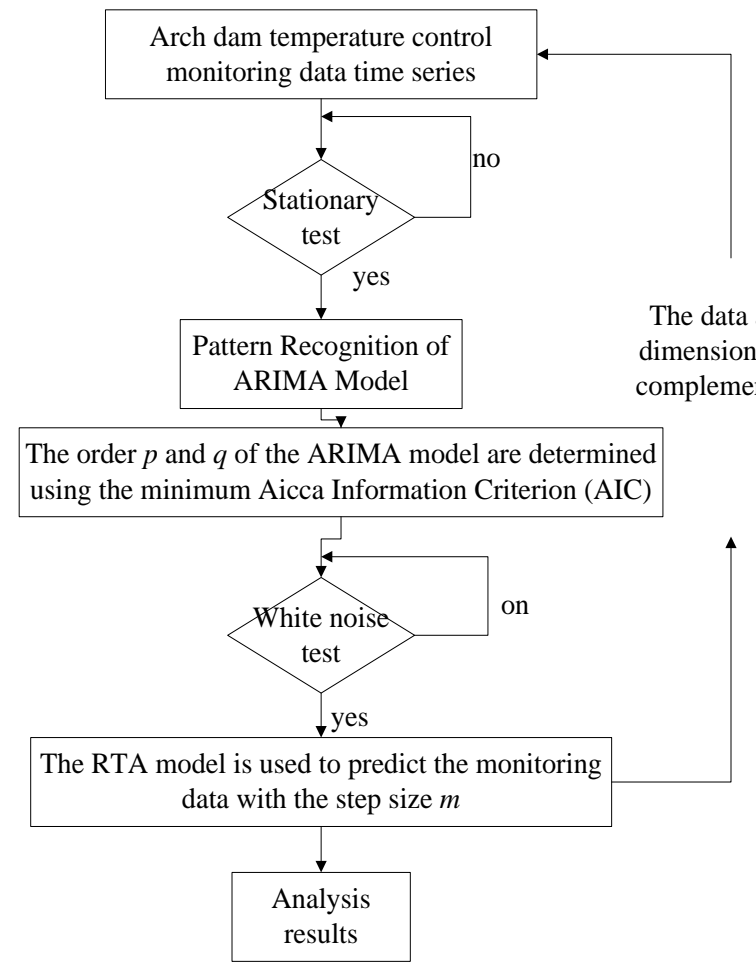

Fig. 1. Flow chart of ARIMA-RTA combination model

\section{ENGINEERING EXAMPLES}

In order to verify the effectiveness of the ARIMA-RTA model's MATLAB program presented in the previous section, the temperature monitoring data of Bailianya arch dam are taken as an example.

Bailianya Dam is located in Huoshan County, Anhui Province. It is a Roller compacted concrete arch dam (RCC arch dam) with a height of 104.6 meters, a crest length of 422.9 meters and a roller compacted concrete volume of 591,000 cubic meters, The dam's height,Arc length,the amount of concrete pouring are all in the forefront of similar dam, and reservoir geological conditions are complex, so Bailianya Dam's the construction difficulty is much higher than the gravity of the arch dam. The dam has been under construction since 2004, the stability of the project has been all aspects of attention. In this paper, the real-time temperature monitoring data of the construction period of 188-K91-01from 2008-3-1 to 2008-5-30 are selected as samples to establish the time series of the monitoring data. The ARIMA model is used to analyze the temperature monitoring data. Model selection of the first 20 monitoring data as the calculation of data, the latter 6 data to test, for the convenience of calculation, to monitor the data of the time has been numbered. To facilitate the calculation, the time to monitor the data has been numbered, $r=1,2, \cdots$. 


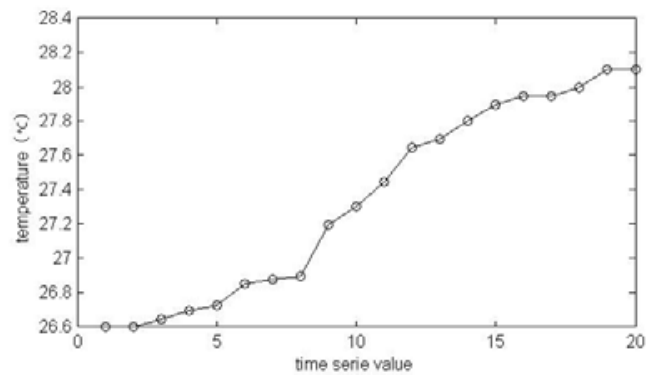

Fig. 2. Time series of arch dam temperature detection

The autocorrelation function (AC) and the partial autocorrelation function (PAC) are used to test the stability of time series of arch dam temperature change. It can be seen that the AC and PAC values are beyond the allowable range and the stationary time series is not satisfied Calculation requirements, the need for differential calculation. AC and PAC coefficients of arch dam temperature monitoring data are shown in Fig.3
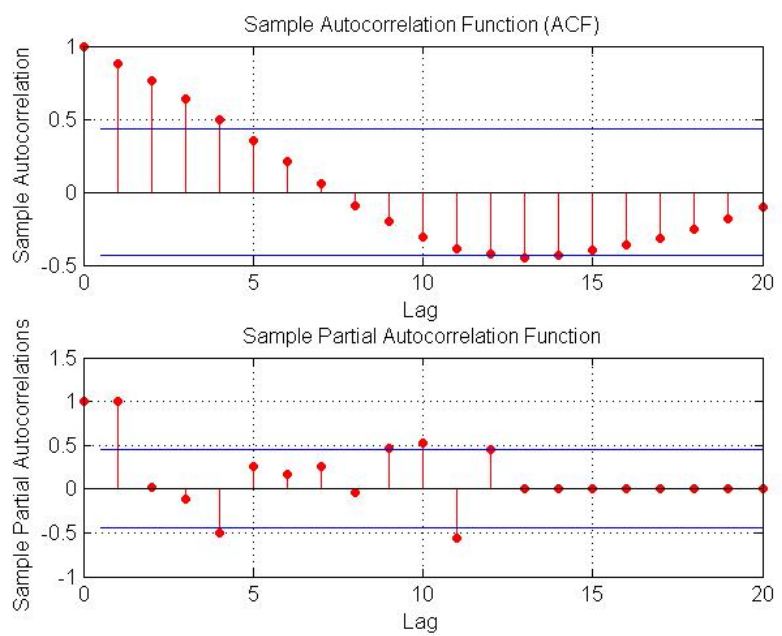

Fig. 3. AC and PAC coefficients of arch dam temperature monitoring data

Through the difference calculation, the primary difference sequence, AC and PAC coefficients of arch dam temperature monitoring data are shown in Fig.4 and Fig.5. It can be seen that the primary difference sequence value of arch dam temperature monitoring data is stable and the AC and PAC coefficients are also basically within the allowable error. The coefficient d in the ARIMA model is set to be 1 .

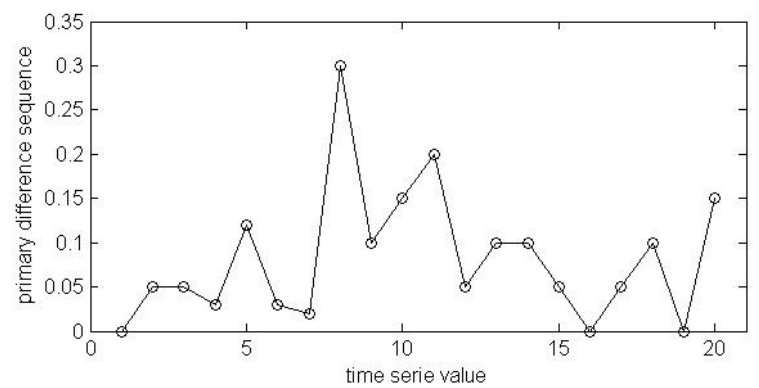

Fig. 4. The primary difference sequence of arch dam temperature monitoring data
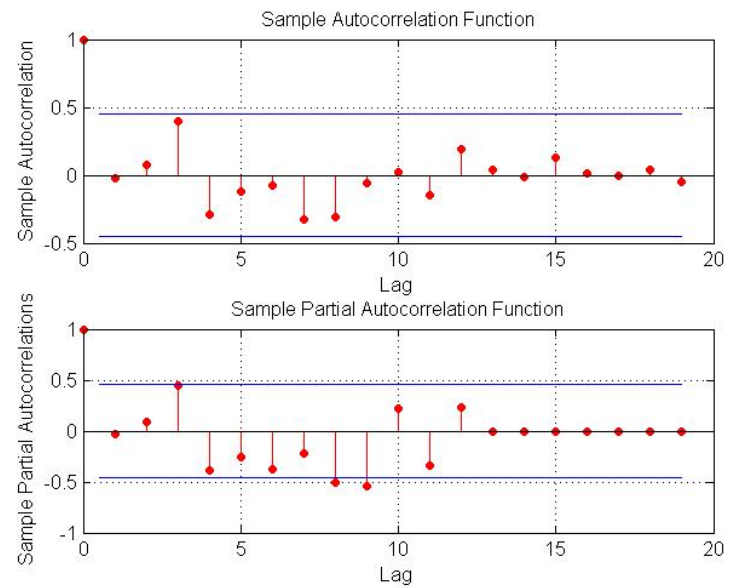

Fig. 5. AC and PAC values of primary differential sequence for arch dam temperature monitoring data

The order of the ARIMA model, $p$ and $q$ is then determined using the minimum Aicca Information Criterion (AIC). It is found that when $p=5$ and $q=3$, the AIC value is the smallest, so the $p$ value of ARIMA model is 5 and the value of $q$ is 3 . The expression of the ARIMA model can be written as $\Phi(B) \nabla x_{i}=\Theta(B) \varepsilon_{i}$ :

where: $\nabla=(1-B)$,

$$
\begin{aligned}
& \Phi(B)=1-0.3195 B-0.2808 B^{2}-0.5614 B^{3}+0.3267 B^{4}-0.00387 B^{5} \\
& \Theta(B)=1-0.5015 B+0.5294 B^{2}-1.015 B^{3}
\end{aligned}
$$

Where $\varepsilon_{i}$ is a white noise sequence

The residual residuals of ARIMA (5, 1, and 3) model and the original time series are shown in Fig. 6, and the fitting residual error of the model is controlled within $2 \mathrm{~mm}$ and the precision is high.

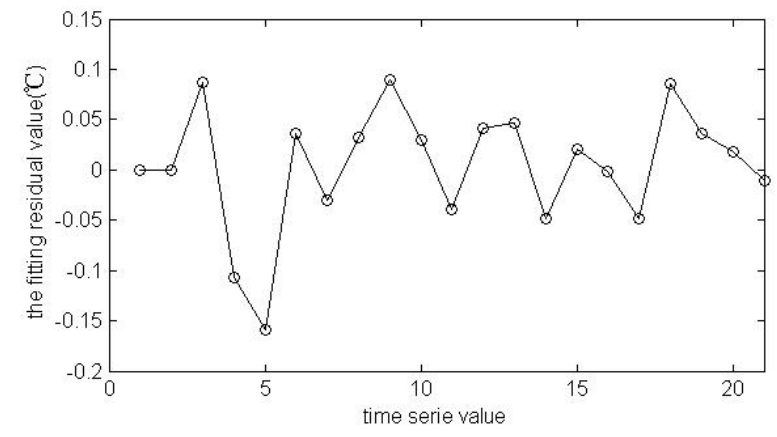

Fig. 6. The fitting residual value of ARIMA model and original monitoring data

The ARIMA (5, 1, and 3) model has higher $p$ and $q$ parameters, which increases the fitting accuracy of the model, but also limits the prediction length. In order to improve the prediction accuracy, we use the ARIMA-RTA combination model to calculate the temperature change of the arch dam in the future six time series by using the isometric recursive cycle calculation of the time series, and the predicted results are compared with the measured values and ARIMA model 
predictions, as shown in Table 1 . It can be seen that the ARIMA-RTA combined model has a significant improvement in prediction accuracy compared with the ARIMA model.

TABLE I.

ARIMA MODEL PREDICTIVE VALUE AND ERROR

\begin{tabular}{cccccc}
\hline sequence & Observations & $\begin{array}{c}\text { ARIMA- } \\
\text { RTA model } \\
\text { predictive } \\
\text { value }\end{array}$ & $\begin{array}{c}\text { Relative } \\
\text { error(\%) }\end{array}$ & $\begin{array}{c}\text { ARIMA } \\
\text { model } \\
\text { predictive } \\
\text { value }\end{array}$ & $\begin{array}{c}\text { Relative } \\
\text { error(\%) }\end{array}$ \\
\hline 21 & 28.25 & 28.302 & $0.18 \%$ & 28.739 & $1.73 \%$ \\
22 & 28.45 & 28.548 & $0.34 \%$ & 29.108 & $2.31 \%$ \\
23 & 28.70 & 28.670 & $-0.10 \%$ & 29.263 & $1.96 \%$ \\
24 & 28.80 & 28.793 & $-0.03 \%$ & 29.513 & $2.48 \%$ \\
25 & 28.95 & 28.914 & $-0.12 \%$ & 29.769 & $2.83 \%$ \\
26 & 29.30 & 29.036 & $-0.90 \%$ & 30.031 & $2.49 \%$ \\
\hline
\end{tabular}

The measured and predicted values in the table are in ${ }^{\circ} \mathrm{C}$

\section{CONCLUSION}

(1) The ARIMA-RTA prediction model is established by combining the ARMA model with the real-time tracking algorithm, and the temperature monitoring data of Bailianya arch dam is taken as an example. The result shows that ARIMA-RTA model can extract the nonlinear dynamic information of the monitoring data through the equaldimension replicating process of the time series. It has good anti-noise ability which can achieve higher prediction accuracy and better prediction length, and provides a new research idea for arch dam temperature monitoring of the forecast analysis.

(2) In the ARIMA-RTA model, the order $p$ and $q$ of the ARIMA model are selected in the fitting prediction process, and the selection of the step size $\mathrm{n}$ by the RTA model has a great influence on the prediction accuracy, so how to optimize these parameters needs further study in the future.

\section{ACKNOWLEDGMENT}

The study was in part supported by the Research Found of Zhejiang Water Resources Department (No. RC1534, RC1548), P.R.China.

\section{REFERENCES}

[1] Wang, Ying Hua, P. Qin, and B. Chen. "Forecasting Model of Monitored Data of Arch Dam's Temperature Based on Improved Variable Dimension Fractal Theory." Journal of Yangtze River Scientific Research Institute 26.12(2009):33-35. (In Chinese)

[2] Su, Huaizhi, P. Qin, and Z. Qin. "A Method for Evaluating Sea Dike Safety." Water Resources Management 27.15(2013):1-14.

[3] Zhong-Ru, W. U., et al. "Theory and method on life diagnosis in important hydraulic engineerings." Chinese Journal of Rock Mechanics \& Engineering (2005). (In Chinese)

[4] Zheng, Dong Jian, and L. I. Feng-Zhen. "Spatiotemporal forecasting of arch dam-abutment deformation considering time-varying effect." Rock \& Soil Mechanics 30.5(2009): 1441-1445..

[5] Qin Peng,Su Huaizhi,Shen Yuejun. "Prediction of seawall settlement based on ARIMA-RTA model". Hydro-Science and Engineering 10.5 (2013),66-70. (In Chinese)

[6] Chen, Huafeng, and S. Chen. "Investment-cash flow sensitivity cannot be a good measure of financial constraints: Evidence from the time series." Journal of Financial Economics 103. 2(2012):393-410. (In Chinese)

[7] Lin, He. "Dynamic Parameter Identification of Steel Frame Structure Based on ARMAX Model and MA Parameter Updating." Journal of Vibration Engineering 15.1(2002):47-51..

[8] Gonzalez, Rafael C., R. E. Woods, and S. L. Eddins. Digital Image processing using MATLAB. Digital image processing/. Pearson/Prentice Hall, 2007: 197-199. 